\title{
Diffusiophoresis in Suspensions of Charged Soft Particles
}

\author{
Wei C. Lin and Huan J. Keh *
}

Department of Chemical Engineering, National Taiwan University, Taipei 10617, Taiwan; r08524053@ntu.edu.tw

* Correspondence: huan@ntu.edu.tw; Tel.: +886-2-33663048

Received: 8 June 2020; Accepted: 27 July 2020; Published: 29 July 2020

\begin{abstract}
The diffusiophoresis in a suspension of charged soft particles in electrolyte solution is analyzed. Each soft particle is composed of a hard core of radius $r_{0}$ and surface charge density $\sigma$ and an adsorbed fluid-penetrable porous shell of thickness $a-r_{0}$ and fixed charge density $Q$. The effect of particle interactions is considered by using a unit cell model. The ionic concentration, electric potential, and fluid velocity distributions in a unit cell are solved as power expansions in $\sigma$ and $Q$, and an explicit formula for the diffusiophoretic velocity of the soft particle is derived from a balance between the hydrodynamic and electrostatic forces exerted on it. This formula is correct to the second orders of $\sigma$ and $Q$ and valid for arbitrary values of $\kappa a, \lambda a, r_{0} / a$, and the particle volume fraction of the suspension, where $\kappa$ is the Debye screening parameter and $\lambda$ is the reciprocal of a length featuring the flow penetration into the porous shell. The effects of the physical characteristics and particle interactions on the diffusiophoresis (including electrophoresis and chemiphoresis) in a suspension of charged soft particles, which become those of hard particles and porous particles in the limits $r_{0}=a$ and $r_{0}=0$, respectively, are significant and complicated.
\end{abstract}

Keywords: diffusiophoresis; electrophoresis; charged soft sphere; arbitrary electric double layer; particle concentration effect

\section{Introduction}

Diffusiophoresis regards the migration of colloidal particles in a fluid solution caused by an imposed solute concentration gradient [1-6] and provides a transport mechanism in many practical applications such as latex film coating [7], autonomous motion of micromotors [8,9], DNA translocation and sequencing [10], colloidal transport in dead-end pores involved in systems of self-regulated drug delivery and enhanced oil recovery [11,12], and particle manipulation and characterization in microfluidic systems [13-16]. For diffusiophoresis of charged particles in electrolyte solutions, the solute-particle interaction is electrostatic and characterized by the Debye screening length $\kappa^{-1}$. Analytical investigations of the diffusiophoretic motion of charged hard particles (impermeable to both the solvent and small ions) under a general imposed electrolyte concentration gradient are mainly restricted to the case of thin electric double layer ( $\kappa a>>1$, where $a$ is the particle radius) $[17,18]$. With the assumption of weak imposed electrolyte concentration gradients, diffusiophoretic motions were also analyzed for a charged hard sphere [19,20], porous sphere (permeable to the ionic solution) [21], soft sphere (a hard core covered by a surface porous layer) [22], and porous spherical shell (microcapsule) [23] with arbitrary values of $k a$.

In real situations of diffusiophoresis, concentrated suspensions of particles may be encountered, and the particle interaction effects are important. To alleviate the complexity of multiple particles, unit cell models are often used to evaluate the particle interaction effects on the mean sedimentation velocity [24-29], average electrophoretic mobility [30-37], and effective electric conductivity [34-38] in suspensions of spherical particles. An agreement between the experimental electrophoretic velocity in 
a suspension of porous aggregates and the relevant cell-model predictions in a broad range of $k a$ was obtained [39].

The diffusiophoretic motions in concentrated suspensions of charged hard spheres of constant zeta potential [40] and porous spheres [41] with arbitrary double-layer thicknesses and particle volume fractions were analyzed using unit cell models. In this article, these studies will be extended to suspensions of charged soft particles. An analytical expression for the diffusiophoretic mobility in the suspension is derived from the balance between the electrostatic and hydrodynamic forces acting on the particles as a power expansion in the fixed-charge densities of the soft spheres.

\section{Electrokinetic Equations}

The surfaces of colloidal particles are generally not hard. For instance, surface layers are purposely formed by adsorbing polymers to make the suspended particles stable against flocculation. Even the surfaces of silica and polystyrene particles are "hairy" with a gel-like polymeric layer extending from the bulk material inside the particle. In particular, the surface of a biological cell or enzyme is not a hard wall, but rather is a permeable rough surface with various appendages ranging from protein molecules on the order of nanometers to cilia on the order of microns. Such particles can be modelled as a soft particle having a central rigid core and an outer porous shell.

Consider the diffusiophoresis of a uniform distribution of charged soft spheres in a solution of a symmetric electrolyte. Each soft sphere of radius $a$ consists of a hard core of radius $r_{0}$ and a porous surface layer of constant thickness $a-r_{0}$. A constant electrolyte concentration gradient $\nabla n^{\infty}$ equal to $\left|\nabla n^{\infty}\right| \mathbf{e}_{z}$ is imposed, where $\mathbf{e}_{z}$ is the unit vector in the $z$ direction, and the diffusiophoretic velocity $U \mathbf{e}_{z}$ of the particles needs to be determined. As shown in Figure 1, we use a unit cell model in which each soft sphere is enveloped by a concentric spherical shell of the fluid with an outer radius $b$ such that the volume fraction of the particles in the suspension $\varphi=(a / b)^{3}$. The origin of the spherical coordinates $(r, \theta, \phi)$ is set at the particle center.

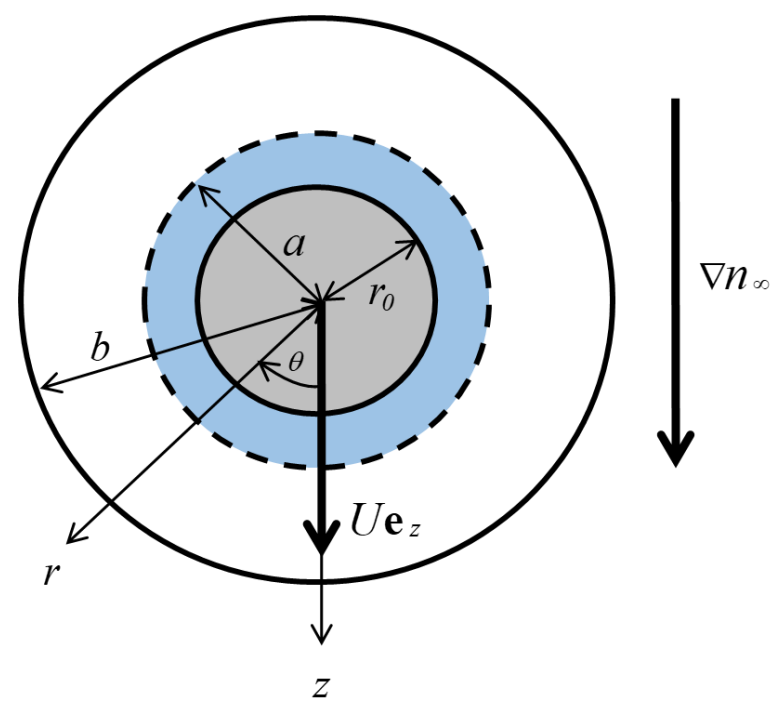

Figure 1. Geometric sketch for the diffusiophoresis of a charged soft sphere in a unit cell.

\subsection{Governing Equations}

The magnitude of $\nabla n^{\infty}$ is assumed to be small (with $a\left|\nabla n^{\infty}\right| / n_{0}^{\infty}<<1$ ) so that the system is only slightly distorted from equilibrium. Thus, the electric potential distribution $\psi(r, \theta)$, the ion 
concentration (number density) distributions $n_{ \pm}(r, \theta)$, and the pressure distribution $p(r, \theta)$ may be written as:

$$
\begin{gathered}
\psi=\psi^{(\mathrm{eq})}+\delta \psi, \\
n_{ \pm}=n_{ \pm}^{(\mathrm{eq})}+\delta n_{ \pm} \\
p=p^{(\mathrm{eq})}+\delta p
\end{gathered}
$$

where $\psi^{(\mathrm{eq})}(r), n_{ \pm}^{(\mathrm{eq})}(r)$, and $p^{(\mathrm{eq})}(r)$ are the equilibrium electric potential, ion concentration, and pressure distributions, respectively, $\delta \psi(r, \theta), \delta n_{ \pm}(r, \theta)$, and $\delta p(r, \theta)$ are the small perturbed quantities, and the subscripts + and - to variables represent the cation and anion, respectively.

Substituting Equation (1) into the Poisson equation, the continuity equation of each ionic species, and the equation of fluid motion (Stokes/Brinkman equation), using the Boltzmann equation to relate the ion concentrations to the electric potential at equilibrium, canceling the equilibrium components, and neglecting the products of the small quantities $\delta n_{ \pm}, \delta \psi$, and fluid velocity field $\mathbf{u}(r, \theta)$, one obtains the following linearized electrokinetic equations [21]:

$$
\begin{gathered}
\nabla^{2} \delta \psi=\frac{Z e n_{0}^{\infty}}{\varepsilon k T}\left[\exp \left(\frac{Z e \psi^{(\mathrm{eq})}}{k T}\right)\left(\delta \mu_{-}+Z e \delta \psi\right)-\exp \left(-\frac{Z e \psi^{(\mathrm{eq})}}{k T}\right)\left(\delta \mu_{+}-Z e \delta \psi\right)\right] \\
\nabla^{2} \delta \mu_{ \pm}= \pm \frac{Z e}{k T}\left\{\nabla \psi^{(\mathrm{eq})} \cdot \nabla \delta \mu_{ \pm}-\frac{k T \nabla \psi^{(\mathrm{eq})} \cdot \mathbf{u}}{D_{ \pm}}\right\} \\
\nabla^{2} \nabla \times \mathbf{u}-h(r) \lambda^{2} \nabla \times \mathbf{u}=-\frac{\varepsilon}{\eta} \nabla \times\left(\nabla^{2} \psi^{(\mathrm{eq})} \nabla \delta \psi+\nabla^{2} \delta \psi \nabla \psi^{(\mathrm{eq})}\right)
\end{gathered}
$$

here, the ionic electrochemical potential energy distributions $\delta \mu_{ \pm}(r, \theta)$ are defined as linear combinations of $\delta \psi$ and $\delta n_{ \pm}$

$$
\delta \mu_{ \pm}=k T \frac{\delta n_{ \pm}}{n_{ \pm}^{(\mathrm{eq})}} \pm Z e \delta \psi
$$

$n_{0}^{\infty}$ is the prescribed electrolyte concentration $n^{\infty}$ at $z=0 ; \eta$ and $\varepsilon$ are the viscosity and permittivity, respectively, of the electrolyte solution; $\lambda$ is the reciprocal of the shielding length for flow penetration into the porous layer; $D_{ \pm}$are the diffusion coefficients of the ionic species; $Z$ is the valence of the symmetric electrolyte; $h(r)$ equals unity if $r_{0}<r<a$, and zero if $a<r<b$.

\subsection{Boundary Conditions}

The boundary conditions at the interface between the hard core and porous layer of the particle as well as at the particle surface are:

$$
\begin{gathered}
r=r_{0}: \mathbf{e}_{r} \cdot \nabla \delta \psi=0, \mathbf{e}_{r} \cdot \nabla \delta \mu_{ \pm}=0, \mathbf{u}=0, \\
r=a: \delta \psi, \nabla \delta \psi, \delta \mu_{ \pm}, \nabla \delta \mu_{ \pm}, \mathbf{u}, \text { and } \mathbf{e}_{r} \cdot \boldsymbol{\tau}, \text { are continuous, }
\end{gathered}
$$

where $\tau$ is the hydrodynamic stress tensor of the fluid and $\mathbf{e}_{r}$ is the unit vector in the $r$ direction. In Equation (7), the continuity requirement of the fluid velocity and stress at the particle surface is physically realistic and mathematically consistent [42] (but the effective viscosity of the fluid inside the porous layer might be smaller than the bulk viscosity if its porosity is small, which is not the case considered here).

The boundary conditions at the outer edge of the cell are:

$$
\begin{gathered}
r=b: \delta \psi=-\frac{k T}{Z e} \beta \alpha \frac{r}{a} \cos \theta \\
\delta \mu_{ \pm}=k T(1 \mp \beta) \alpha \frac{r}{a} \cos \theta
\end{gathered}
$$




$$
u_{r}=-U \cos \theta
$$

for the Happel model:

$$
\tau_{r \theta}=\eta\left[r \frac{\partial}{\partial r}\left(\frac{u_{\theta}}{r}\right)+\frac{1}{r} \frac{\partial u_{r}}{\partial \theta}\right]=0
$$

for the Kuwabara model:

$$
(\nabla \times \mathbf{u})_{\phi}=\frac{1}{r} \frac{\partial}{\partial r}\left(r u_{\theta}\right)-\frac{1}{r} \frac{\partial u_{r}}{\partial \theta}=0
$$

where $\beta=\left(D_{+}-D_{-}\right) /\left(D_{+}+D_{-}\right)$(for a binary, symmetric electrolyte) and $\alpha=a\left|\nabla n^{\infty}\right| / n_{0}^{\infty}$. Equations (6) and (10) take a reference frame traveling with the particle, while Equation (8) denotes the induced electric potential arising from the applied electrolyte gradient with different cation and anion mobilities [1,2]. The Happel model assumes that the radial velocity relative to the bulk flow and the shear stress of the fluid on the outer boundary of the cell are zero, while the Kuwabara model assumes that this radial velocity and the vorticity of the fluid are zero there. The Happel model has an advantage over the Kuwabara model in that the former does not require an exchange of mechanical energy between the cell and the environment [43].

\section{Solution for the Diffusiophoretic Velocity}

\subsection{Equilibrium Electric Potential}

For a soft sphere with a uniform surface charge density $\sigma$ of its hard core and a constant space charge density $Q$ of its porous surface layer in a unit cell, the equilibrium potential distribution $\psi^{(\mathrm{eq})}(r)$ can be obtained as:

$$
\psi^{(\mathrm{eq})}=\psi_{\mathrm{eq} 01} \bar{\sigma}+\psi_{\mathrm{eq} 10} \bar{Q}+\mathrm{O}\left(\bar{\sigma}^{3}, \bar{\sigma}^{2} \bar{Q}, \bar{\sigma}_{\bar{Q}}^{2}, \bar{Q}^{3}\right)
$$

where $\bar{\sigma}=Z e \sigma / \varepsilon \kappa k T, \bar{Q}=Z e Q / \varepsilon \kappa^{2} k T, \psi_{\text {eq01 }}(r)$ and $\psi_{\text {eq10 }}(r)$ are available in the literature [24] and $\kappa=\left[2 Z^{2} e^{2} n_{0}^{\infty} / \varepsilon k T\right]^{1 / 2}$ is the Debye screening parameter. The expression in Equation (13) for $\psi^{(\mathrm{eq})}$ as a power series in $\bar{\sigma}$ and $\bar{Q}$ up to $\mathrm{O}(\bar{\sigma}, \bar{Q})$ is valid for small values of the electric potential (the Debye-Hückel approximation).

\subsection{Solution to Electrokinetic Equations}

The quantities $u_{r}, u_{\theta}$ (the $r$ and $\theta$ components of $\mathbf{u}$ ), $\delta p, \delta \psi$, and $\delta \mu_{ \pm}$satisfying Equations (2)-(4) and the equation of continuity $\nabla \cdot \mathbf{u}=0$ can be solved in terms of $\psi^{(\mathrm{eq})}$ given by Equation (13) and the expansion form of the particle velocity $U$,

$$
U=U_{01} \bar{\sigma}+U_{10} \bar{Q}+U_{02} \bar{\sigma}^{2}+U_{11} \bar{\sigma} \bar{Q}+U_{20} \bar{Q}^{2}+\ldots
$$

with the results:

$$
\begin{gathered}
\delta \psi=\frac{k T}{Z e} \alpha\left[-\beta F_{\psi 00}(r)+F_{\psi 01}(r) \bar{\sigma}+F_{\psi 10}(r) \bar{Q}+\ldots\right] \cos \theta \\
\delta \mu_{ \pm}=k T(1 \mp \beta) \alpha\left[F_{\mu 00}(r) \mp F_{\mu 01}(r) \bar{\sigma} \mp F_{\mu 10}(r) \bar{Q}+\ldots\right] \cos \theta \\
u_{r}=\left\{\left[U_{01} F_{00 r}(r)-\frac{k T}{\eta a^{2}} \beta \alpha F_{01 r}(r)\right] \bar{\sigma}+\left[U_{10} F_{00 r}(r)-\frac{k T}{\eta a^{2}} \beta \alpha F_{10 r}(r)\right] \bar{Q}\right. \\
+\left[U_{02} F_{00 r}(r)+\frac{k T}{\eta a^{2}} \alpha F_{02 r}(r)\right] \bar{\sigma}^{2}+\left[U_{11} F_{00 r}(r)+\frac{k T}{\eta a^{2}} \alpha F_{11 r}(r)\right] \bar{\sigma} \bar{Q} \\
\left.+\left[U_{20} F_{00 r}(r)+\frac{k T}{\eta a^{2}} \alpha F_{20 r}(r)\right] \bar{Q}^{2}+\ldots\right\} \cos \theta \\
u_{\theta}=-\frac{\partial\left(r^{2} u_{r}\right)}{2 r \partial r} \tan \theta
\end{gathered}
$$




$$
\begin{gathered}
\delta p=\frac{\eta}{a}\left\{\left[U_{01} F_{p 00}(r)-\frac{k T}{\eta a^{a^{2}}} \beta \alpha F_{p 01}(r)-\frac{\varepsilon \kappa^{2} a k T}{\eta Z e} \beta \alpha \psi_{\mathrm{eq} 01}(r) F_{\psi 00}(r)\right] \bar{\sigma}\right. \\
+\left[U_{10} F_{p 00}(r)-\frac{k T}{\eta a^{2}} \beta \alpha F_{p 10}(r)-\frac{\varepsilon \kappa^{2} a k T}{\eta Z e} \beta \alpha \psi_{\mathrm{eq} 10}(r) F_{\psi 00}(r)\right] \bar{Q} \\
+\left[U_{02} F_{p 00}(r)+\frac{k T}{\eta a^{2}} \alpha F_{p 02}(r)+\frac{\varepsilon \kappa^{2} a k T}{\eta Z e} \alpha \psi_{\mathrm{eq} 10}(r) F_{\psi 10}(r)\right] \bar{\sigma}^{2} \\
+\left[U_{11} F_{p 00}(r)+\frac{k T}{\eta a^{2}} \alpha F_{p 11}(r)+\frac{\varepsilon \kappa^{2} a k T}{\eta Z e} \alpha\left(\psi_{\mathrm{eq} 01}(r) F_{\psi 10}(r)+\psi_{\mathrm{eq} 10}(r) F_{\psi 01}(r)\right)\right] \bar{\sigma} \bar{Q} \\
\left.+\left[U_{20} F_{p 00}(r)+\frac{k T}{\eta a^{2}} \alpha F_{p 20}(r)+\frac{\varepsilon \kappa^{2} a k T}{\eta Z e} \alpha \psi_{\mathrm{eq} 01}(r) F_{\psi 01}(r)\right] \bar{Q}^{2}+\ldots\right\} \cos \theta
\end{gathered}
$$

where, $F_{00 r}(r), F_{p 00}(r), F_{i j r}(r), F_{p i j}(r), F_{\mu 00}(r), F_{\psi 00}(r), F_{\mu 01}(r), F_{\mu 10}(r), F_{\psi 01}(r)$, and $F_{\psi 10}(r)$ are dimensionless functions given by Equations (A1)-(A8) and (A20)-(A23) in the Appendix A, the particle velocities $U_{i j}$ are to be determined from a force balance, and the set $(i, j)$ equals $(0,1),(1,0)$, $(0,2),(1,1)$, and $(2,0)$. The zeroth-order terms of $\mathbf{u}, \delta p$, and $U$ disappear because the electrolyte solution around an uncharged particle will not move by imposing an electrolyte concentration gradient if only the electrostatic interaction is considered. To solve the small quantities $\delta \psi, \delta \mu_{ \pm}, \mathbf{u}$, and $\delta p$ in terms of the diffusiophoretic velocity $U$, these variables can be written as perturbation expansions in powers of $\bar{\sigma}$ and $\bar{Q}$. Substituting these expansions and $\psi^{(\mathrm{eq})}$ into the governing Equations (2)-(4) and boundary conditions (6)-(12) and equating like powers of $\bar{\sigma}$ and $\bar{Q}$ on both sides of the respective equations, we obtain a group of linear differential equations and boundary conditions of orders 0,1 , and 2 . These perturbation equations can be analytically solved, and the results for the $\rho$ and $\phi$ components of $\mathbf{u}, \delta p$ (to the order of $\bar{\sigma}^{2}, \bar{\sigma} \bar{Q}$, and $\bar{Q}^{2}$ ), $\delta \mu_{ \pm}$, and $\delta \psi$ (to the order of $\bar{\sigma}$ and $\bar{Q}$ ) in Equations (15)-(19) are obtained.

\subsection{Forces Exerted on the Particle}

The total force exerted on a soft particle is the sum of the electrostatic and hydrodynamic forces. The electrostatic force is determined as an integral of the electric force density over the region $a \leq r \leq b$ (since the overall unit cell is electrically neutral and the electrostatic force acting on its outer boundary $r=b$ vanishes), with the result

$$
\begin{gathered}
\mathbf{F}_{\mathrm{e}}=4 \pi \frac{k T}{a} \alpha\left\{-\beta\left[\frac{\varepsilon \kappa^{2} a^{3}}{3 Z e} \psi_{\mathrm{eq} 01}(a) F_{\psi 00}(a)-\frac{\varepsilon \kappa^{2} a b^{2}}{3 Z e} \psi_{\mathrm{eq} 01}(b) F_{\psi 00}(b)+J_{01}^{(3)}(b)\right] \bar{\sigma}\right. \\
-\beta\left[\frac{\varepsilon \kappa^{2} a^{3}}{3 Z e} \psi_{\mathrm{eq} 10}(a) F_{\psi 00}(a)-\frac{\varepsilon \kappa^{2} a b^{2}}{3 Z e} \psi_{\mathrm{eq} 10}(b) F_{\psi 00}(b)+J_{10}^{(3)}(b)\right] \bar{Q} \\
+\left[\frac{\varepsilon \kappa^{2} a^{3}}{3 Z e} \psi_{\mathrm{eq} 01}(a) F_{\psi 01}(a)-\frac{\varepsilon \kappa^{2} a b^{2}}{3 Z e} \psi_{\mathrm{eq} 01}(b) F_{\psi 01}(b)+J_{02}^{(3)}(b)\right] \bar{\sigma}^{2} \\
+\left[\frac{\varepsilon \kappa^{2} a^{3}}{3 Z e}\left\{\psi_{\mathrm{eq} 01}(a) F_{\psi 10}(a)+\psi_{\mathrm{eq} 10}(a) F_{\psi 01}(a)\right\}\right. \\
\left.-\frac{\varepsilon \kappa^{2} a b^{2}}{3 Z e}\left\{\psi_{\mathrm{eq} 01}(b) F_{\psi 10}(b)+\psi_{\mathrm{eq} 10}(b) F_{\psi 01}(b)\right\}+J_{11}^{(3)}(b)\right] \bar{\sigma} \bar{Q} \\
\left.+\left[\frac{\varepsilon \kappa^{2} a^{3}}{3 Z e} \psi_{\mathrm{eq} 10}(a) F_{\psi 10}(a)-\frac{\varepsilon \kappa^{2} a b^{2}}{3 Z e} \psi_{\mathrm{eq} 10}(b) F_{\psi 10}(b)+J_{20}^{(3)}(b)\right] \bar{Q}^{2}+\ldots\right\} \mathbf{e}_{z}
\end{gathered}
$$

where the functions $J_{i j}^{(3)}(r)$ are defined by Equation (A11). 
The hydrodynamic force acting on the soft particle may be obtained as an integral of the hydrodynamic stress over the particle surface $(r=a)$, with the result

$$
\begin{gathered}
\mathbf{F}_{\mathrm{h}}=-4 \pi\left\{\left[\eta a C_{002} U_{01}-\frac{k T}{a} \beta \alpha C_{012}-\frac{\varepsilon(\kappa a)^{2}}{3} \frac{k T}{Z e} \beta \alpha \psi_{\mathrm{eq} 01}(a) F_{\psi 00}(a)\right] \bar{\sigma}\right. \\
+\left[\eta a C_{002} U_{10}-\frac{k T}{a} \beta \alpha C_{102}-\frac{\varepsilon(\kappa a)^{2}}{3} \frac{k T}{Z e} \beta \alpha \psi_{\mathrm{eq} 10}(a) F_{\psi 00}(a)\right] \bar{Q} \\
+\left[\eta a C_{002} U_{02}+\frac{k T}{a} \alpha C_{022}+\frac{\varepsilon(\kappa a)^{2}}{3} \frac{k T}{Z e} \alpha \psi_{\mathrm{eq} 01}(a) F_{\psi 01}(a)\right] \bar{\sigma}^{2} \\
+\left[\eta a C_{002} U_{11}+\frac{k T}{a} \alpha C_{112}+\frac{\varepsilon(\kappa a)^{2}}{3} \frac{k T}{Z e} \alpha\left\{\psi_{\mathrm{eq} 01}(a) F_{\psi 10}(a)+\psi_{\mathrm{eq} 10}(a) F_{\psi 01}(a)\right\}\right] \bar{\sigma} \bar{Q} \\
\left.+\left[\eta a C_{002} U_{20}+\frac{k T}{a} \alpha C_{202}+\frac{\varepsilon(\kappa a)^{2}}{3} \frac{k T}{Z e} \alpha \psi_{\mathrm{eq} 10}(a) F_{\psi 10}(a)\right] \bar{Q}^{2}+\ldots\right\} \mathbf{e}_{z}
\end{gathered}
$$

where the coefficients $C_{002}$ and $C_{i j 2}$ are given in Equations (A1) and (A5). Like the fluid flow field in Equations (17)-(19), the zeroth-order terms of the electrostatic and hydrodynamic forces vanish.

\subsection{Velocity of the Particle}

Applying the constraint that the total force acting on the soft sphere is zero at the steady state to the summation of Equations (20) and (21), we obtain:

$$
U_{i j}=\frac{\varepsilon \beta^{(2-i-j)} \alpha}{\eta a}\left(\frac{k T}{Z e}\right)^{2}(\kappa a)^{2 i+j} H_{i j}
$$

where, $H_{i j}$ are dimensionless functions of the electrokinetic radius $\kappa a$, radius ratio $r_{0} / a$, hydrodynamic resistance parameter $\lambda a$, and volume fraction $\varphi$ of the particle defined by:

$$
\begin{gathered}
H_{i j}=\frac{(-1)^{i+j-1}(Z e)^{2}}{C_{002}(\kappa a)^{2 i+j} \varepsilon a k T}\left\{C_{i j 2}-J_{i j}^{(3)}(b)+\frac{\varepsilon \kappa^{2} a b^{2}}{3 Z e}\left[\left(1-\delta_{i j}\right) \psi_{\text {eqst }}(b) F_{\psi u v}(b)\right.\right. \\
\left.\left.+\delta_{i j}\left\{\psi_{\text {eq } 01}(b) F_{\psi 10}(b)+\psi_{\text {eq10 }}(b) F_{\psi 01}(b)\right\}\right]\right\}
\end{gathered}
$$

where the functions $J_{i j}^{(3)}(r)$ are defined by Equation (A11), $\delta_{i j}$ is the Kronecker delta which equals unity if $i=j$ but vanishes otherwise, $s=i /(i+j), t=j /(i+j), u=(i-1) s$, and $v=(j-1) t$. The diffusiophoretic velocity of the charged soft sphere is obtained in Equations (14), (22), and (23).

When the hard core of each soft particle vanishes $\left(r_{0}=0\right)$, it reduces to an entirely porous particle of radius $a$ and fixed charge density $Q$, the dimensionless mobility coefficients $H_{01}, H_{02}$, and $H_{11}$ (or $U_{01}, U_{02}$, and $U_{11}$ ) are trivial, and $H_{10}$ and $H_{20}$ given by Equation (23) are identical to those available in the literature [41].

\section{Results and Discussion}

The mean diffusiophoretic mobility of identical charged soft spheres suspended in a symmetric electrolyte solution can be determined to the second orders $\sigma^{2}, \sigma Q$, and $Q^{2}$ of their fixed charge densities using Equations (14), (22), and (23). In this section, we will consider the mobility in a suspension of hard spherical particles with constant surface charge density $\sigma$ first and results for a suspension of soft spheres are then presented.

\subsection{Suspension of Hard Spheres}

For a suspension of hard spheres with radius $a=r_{0}$, the dimensionless mobility coefficients $H_{10}, H_{20}$, and $H_{11}$ (or $U_{10}, U_{20}$, and $U_{11}$ ) become trivial, and $H_{01}$ and $H_{02}$ calculated from Equation (23) are functions of the electrokinetic radius $\kappa a$ and volume fraction $\varphi\left(=a^{3} / b^{3}\right)$ of the particles. In 
Figure 2, results of the electrophoretic mobility coefficient $H_{01}$ for a suspension of hard spheres with constant surface charge density $\sigma$ are presented up to $\varphi=0.74$, the limit for an assemblage of identical spheres [30]. Evidently, $H_{01}$ is always positive; thus the direction of the particle movement contributed by electrophoresis is determined by the sign of the product of the surface charge density $\sigma$ and the ionic diffusivity parameter $\beta$ (along the electrolyte concentration gradient if $\beta \sigma>0$ and against it if $\beta \sigma<0$ ). For a given value of $\varphi$, the value of $H_{01}$ is a monotonic decreasing function of $\kappa a$ from a positive constant at $\kappa a=0$ to zero as $\kappa a \rightarrow \infty$. For a given value of $\kappa a$, the value of $H_{01}$ decreases monotonically with an increase in $\varphi$ from a positive constant at $\varphi=0$ and the particle interaction effect on the electrophoretic mobility can be significant. As expected, $H_{01}=2 / 3$ (the Hückel result for electrophoresis) in the particular case of $\varphi=0$ and $\kappa a=0$. The Happel model always predicts a slightly greater value (weaker particle interaction effect) for the electrophoretic velocity than the Kuwabara model does. This occurs because the zero-vorticity Kuwabara model yields larger energy dissipation in the cell than that due to the particle drag alone, owing to the additional work done by the stresses at the outer boundary [43].
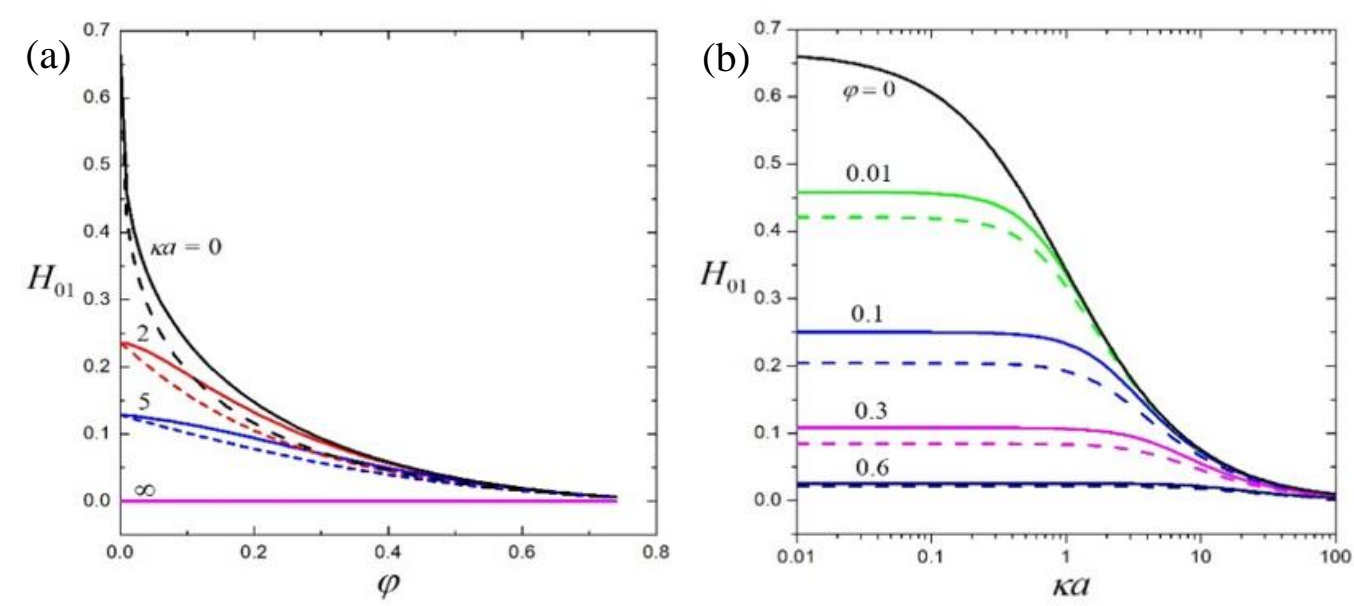

Figure 2. The electrophoretic mobility coefficient $H_{01}$ for a suspension of hard spheres versus the parameters (a) $\varphi$ and (b) $\kappa a$. The solid and dashed curves represent the calculations for the Happel and Kuwabara models, respectively, and $H_{01}=2 / 3$ in the particular case of $\varphi=0$ and $\kappa a=0$.

The results for the chemiphoretic mobility coefficient $\mathrm{H}_{02}$ of a suspension of hard spheres with constant surface charge density $\sigma$ are plotted versus the parameters $\kappa a$ and $\varphi$ in Figure 3. The value of $H_{02}$ is always positive (the chemiphoresis is directed along the electrolyte concentration gradient) and not necessary a monotonic function of $\varphi$ or $\kappa a$, keeping the other unchanged. For a given value of $\kappa a$, the value of $H_{02}$ first increases with an increase in $\varphi$ from a constant at $\varphi=0$, reaches a maximum, and then decreases with a further increase in $\varphi$; the particle interaction effect on the chemiphoretic mobility can also be significant. For relatively concentrated suspensions (say, $\varphi \geq 10^{-4}$ ), $H_{02}$ is a monotonic decreasing function of $\kappa a$ from a constant at $\kappa a=0$ to zero as $\kappa a \rightarrow \infty$. For the particular case of $\varphi=0$, the value of $H_{02}$ first increases with an increase in $\kappa a$ from zero at $\kappa a=0$, reaches a maximum, and then decreases with a further increase in $\kappa a$ to zero as $\kappa a \rightarrow \infty$. The Happel model also predicts a slightly greater value for the chemiphoretic velocity than the Kuwabara model does. 

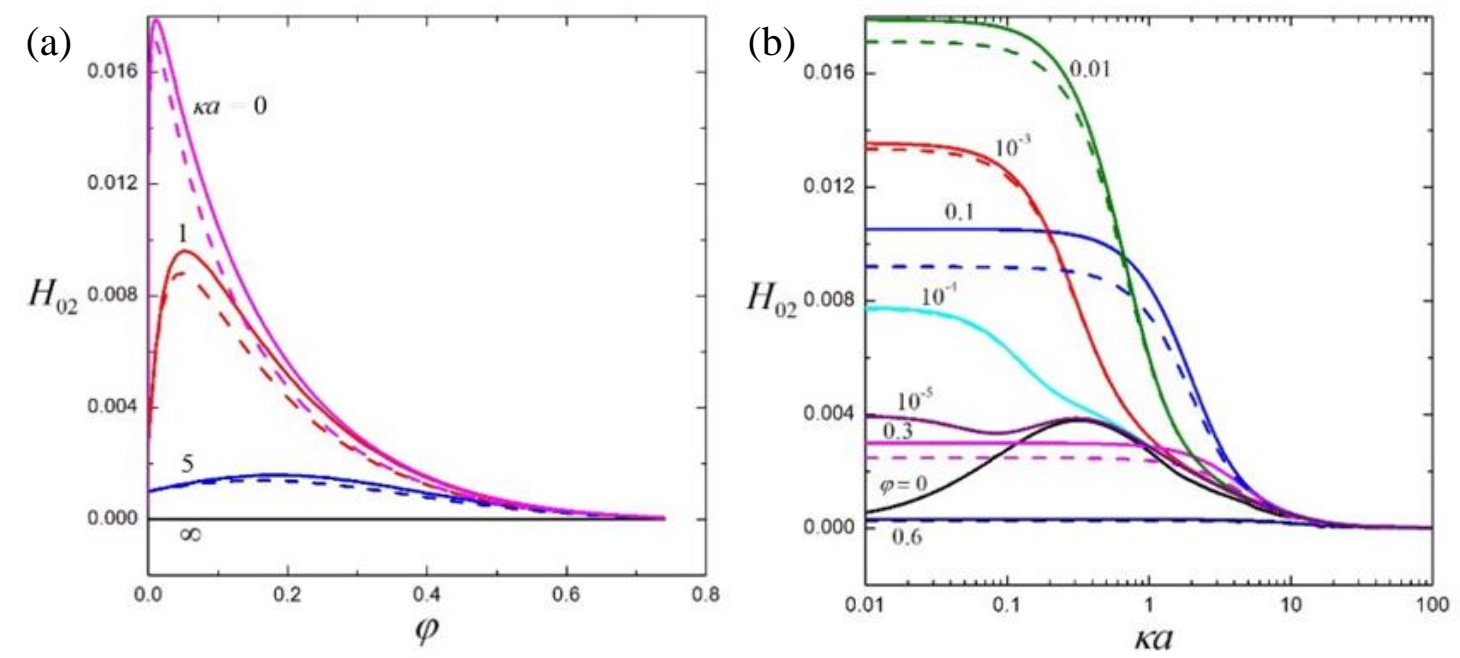

Figure 3. The chemiphoretic mobility coefficient $H_{02}$ for a suspension of hard spheres versus the parameters (a) $\varphi$ and (b) $\kappa a$. The solid and dashed curves represent the calculations for the Happel and Kuwabara models, respectively.

The diffusiophoretic velocity $U$ in Equation (14) for a suspension of hard spheres normalized by the characteristic value $U^{*}=(k T / Z e)^{2} \varepsilon \alpha / \eta a$ as a function of the scaled surface charge density $\kappa a \bar{\sigma}=a Z e \sigma / \varepsilon k T$ calculated for the Happel model at a typical value of $\varphi(\varphi=0.1)$ and various values of $\kappa a$ is plotted in Figure 4. Figure 4a shows the case that the diffusiophoresis is due to the chemiphoresis entirely (the anion and cation of the electrolyte have the same diffusion coefficient or $\beta=0$ ). As expected, the reduced diffusiophoretic mobility $U / U^{*}$ is an even function of the surface charge density and increases monotonically with an increase in $\kappa a|\bar{\sigma}|$ for specified values of $\kappa a$ and $\varphi$. For a relatively concentrated suspension (say, $\varphi \geq 10^{-4}$ ) with a given value of $\kappa a|\bar{\sigma}|, U / U^{*}$ decreases with an increase in $\kappa a$ to zero as $\kappa a \rightarrow \infty$. There is no chemiphoresis of the particles for the particular case of $\kappa a \bar{\sigma}=0$ ( or $\sigma=0$ ). The reduced diffusiophoretic mobility $U / U^{*}$ of the particles for a typical case that the cation and anion have different diffusivities $(\beta=-0.2)$ is plotted in Figure $4 b$, where both electrophoresis and chemiphoresis contribute to the diffusiophoresis. In this case, for constant values of $\kappa a$ and $\varphi$, $U / U^{*}$ is not necessary a monotonic function of $k a \bar{\sigma}$ and the particles may reverse direction of their diffusiophoretic velocity twice with the variation of their surface charge density due to the competition between chemiphoretic and electrophoretic contributions. Note that the cases of $\beta=-0.2$ and $\beta=0$ may denote the aqueous solutions of $\mathrm{NaCl}\left(\mathrm{NaBr}, \mathrm{NaI}, \mathrm{NaNO}_{3}, \mathrm{CaSO}_{4}\right)$ and $\mathrm{KCl}\left(\mathrm{KBr}, \mathrm{KI}, \mathrm{KNO}_{3}\right.$, $\mathrm{NH}_{4} \mathrm{Cl}$ ), respectively. 

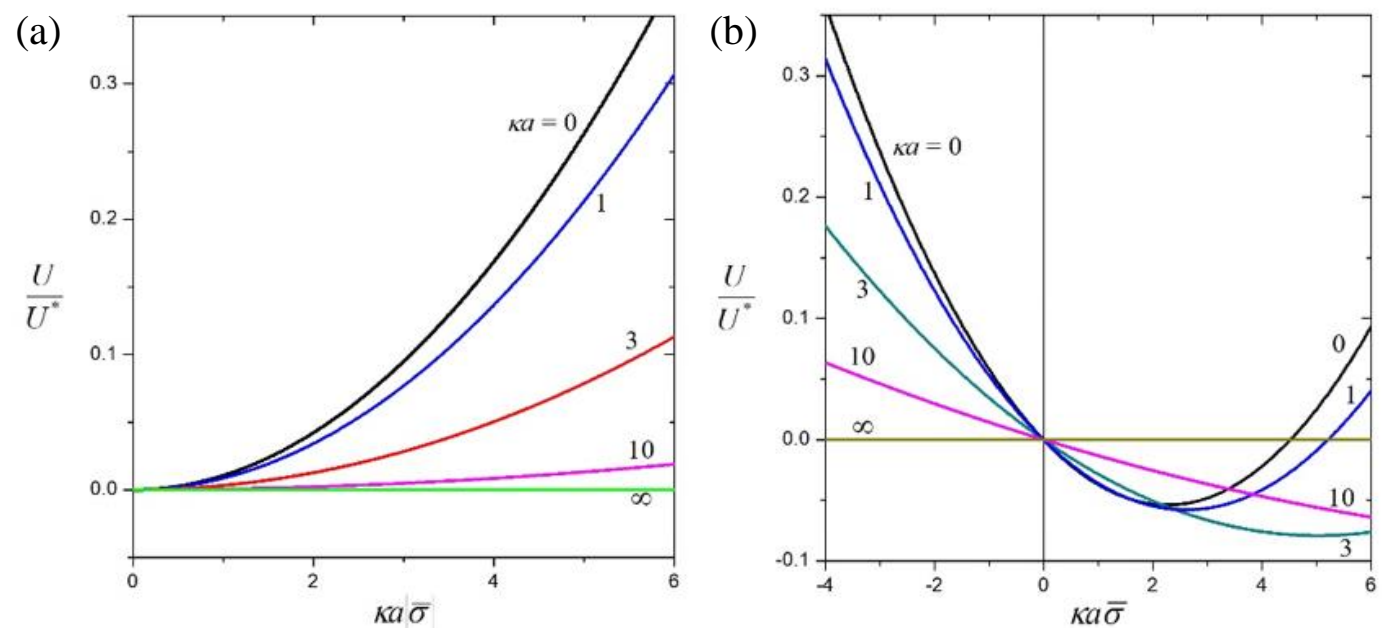

Figure 4. The reduced diffusiophoretic mobility $U / U^{*}$ for a suspension of hard spheres versus the dimensionless surface charge density $\kappa a \bar{\sigma}$ with $\varphi=0.1$ and various values of $\kappa a$ calculated for the Happel model: (a) $\beta=0$; (b) $\beta=-0.2$.

\subsection{Suspension of Soft Spheres}

For a suspension of soft spheres, the diffusiophoretic mobility coefficients $H_{01}, H_{10}, H_{02}, H_{11}$, and $H_{20}$ calculated from Equation (23) are plotted in Figures 5-9, respectively, for various values of the electrokinetic particle radius $\kappa a$, core-to-particle radius ratio $r_{0} / a$, porous-layer shielding parameter $\lambda a$, and particle volume fraction $\varphi$. In general, these mobility coefficients are positive, decreasing functions of $\kappa a$, decreasing functions of $\lambda a$, and slightly greater as predicted by the Happel model than the Kuwabara model.
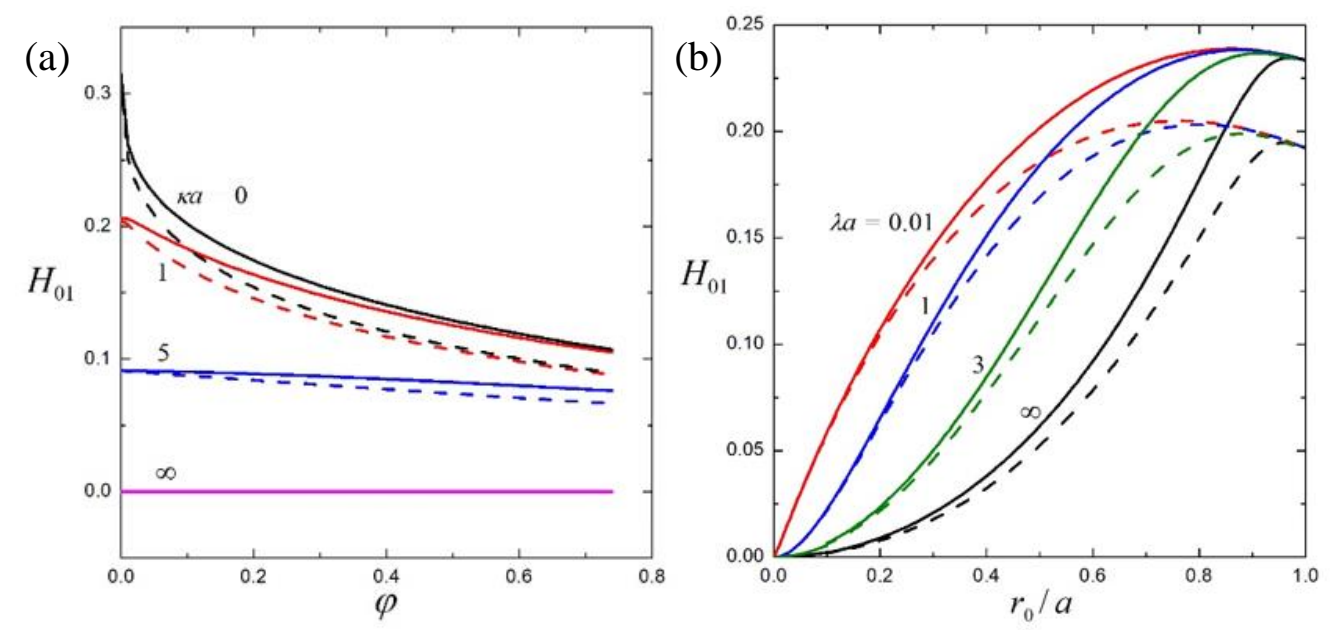

Figure 5. The electrophoretic mobility coefficient $H_{01}$ for a suspension of soft spheres: (a) $\lambda a=1$ and $r_{0} / a=0.5 ;(\mathbf{b}) \kappa a=1$ and $\varphi=0.1$. The solid and dashed curves represent the calculations for the Happel and Kuwabara models, respectively. 

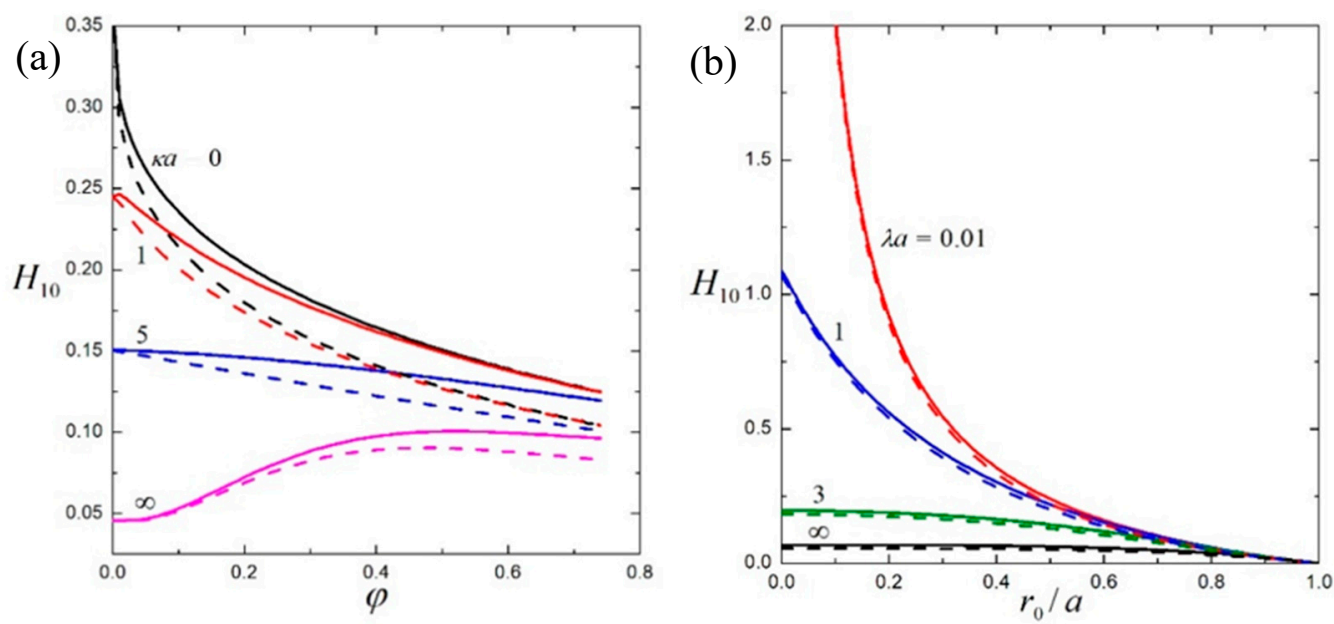

Figure 6. The electrophoretic mobility coefficient $H_{10}$ for a suspension of soft spheres: (a) $\lambda a=1$ and $r_{0} / a=0.5$; (b) $\kappa a=1$ and $\varphi=0.1$. The solid and dashed curves represent the calculations for the Happel and Kuwabara models, respectively.

(a)

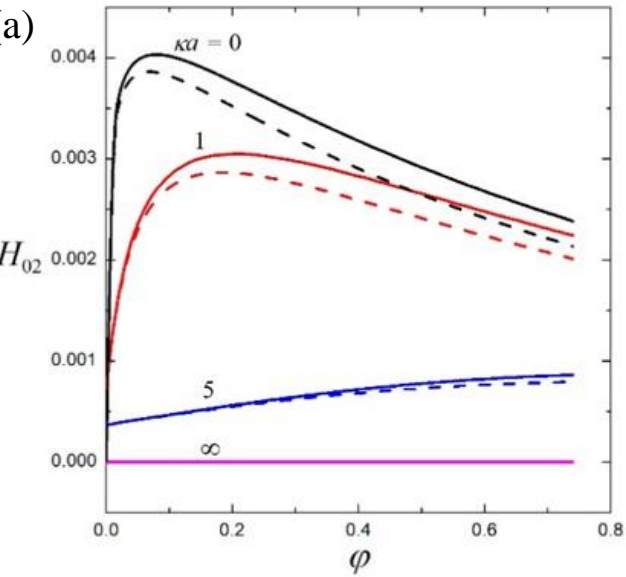

(b)

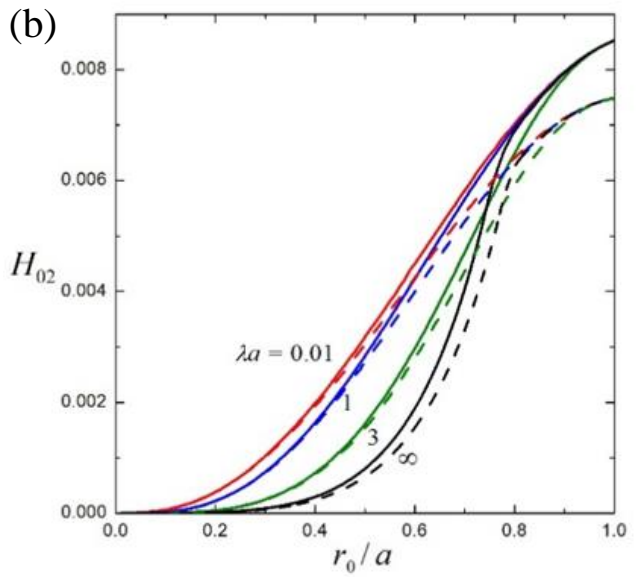

Figure 7. The chemiphoretic mobility coefficient $H_{02}$ for a suspension of soft spheres: (a) $\lambda a=1$ and $r_{0} / a=0.5$; (b) $\kappa a=1$ and $\varphi=0.1$. The solid and dashed curves represent the calculations for the Happel and Kuwabara models, respectively.

(a)

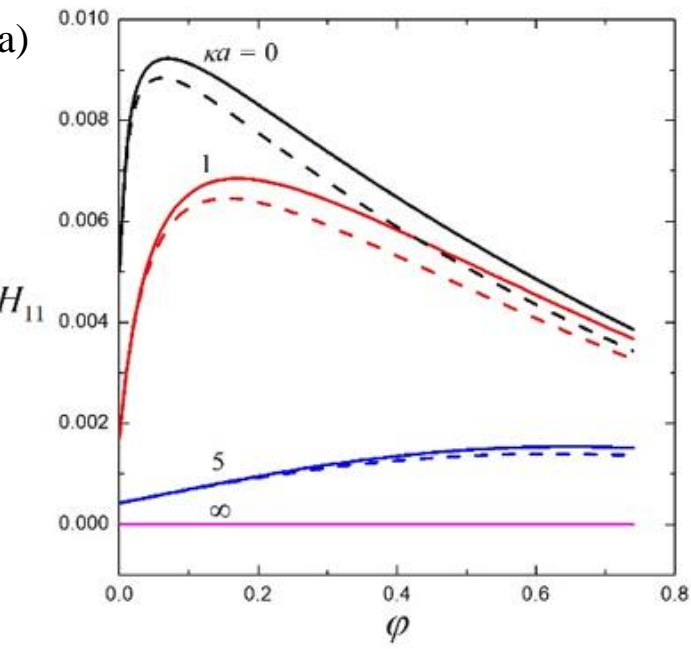

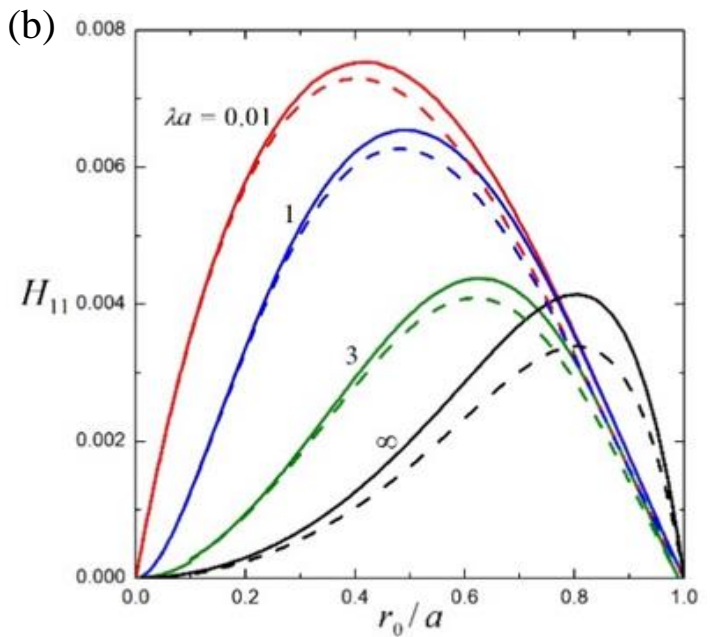

Figure 8. The chemiphoretic mobility coefficient $H_{11}$ for a suspension of soft spheres: (a) $\lambda a=1$ and $r_{0} / a=0.5$; (b) $\kappa a=1$ and $\varphi=0.1$. The solid and dashed curves represent the calculations for the Happel and Kuwabara models, respectively. 

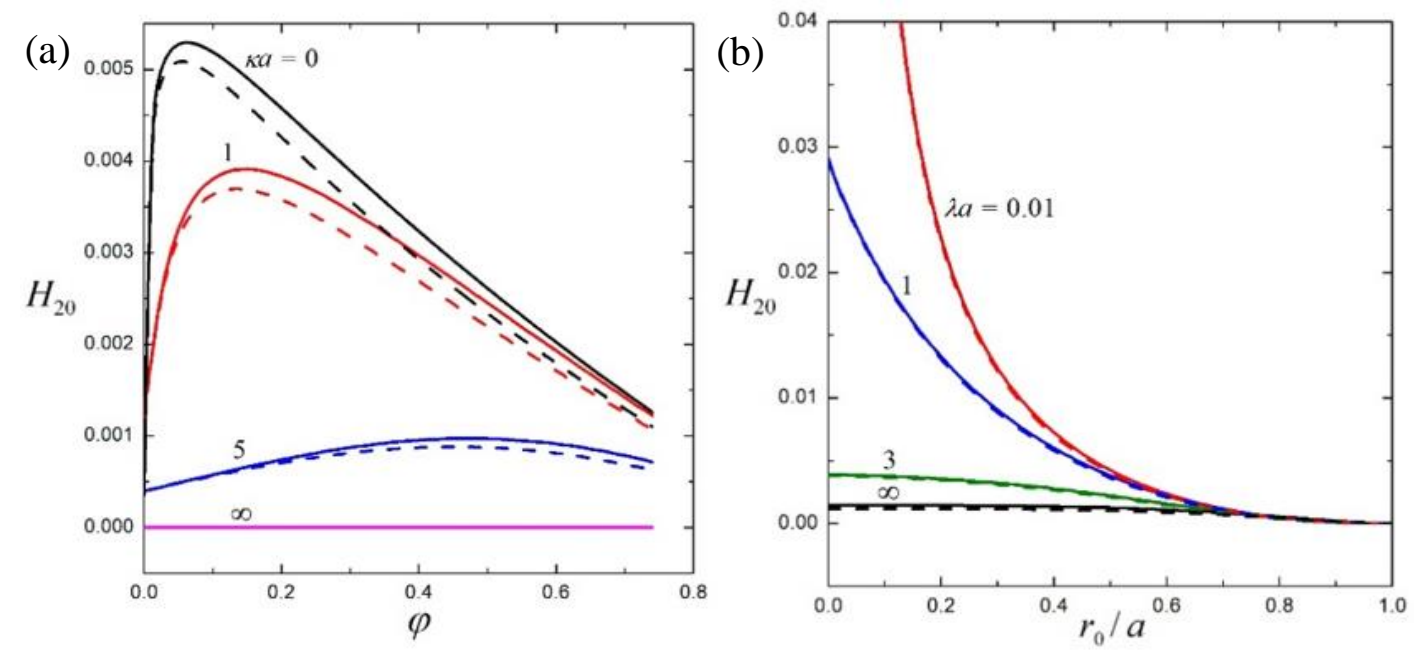

Figure 9. The chemiphoretic mobility coefficient $H_{20}$ for a suspension of soft spheres: (a) $\lambda a=1$ and $r_{0} / a=0.5$; (b) $\kappa a=1$ and $\varphi=0.1$. The solid and dashed curves represent the calculations for the Happel and Kuwabara models, respectively.

Figures 5 and 6 indicate that, for fixed values of $r_{0} / a, k a$, and $\lambda a$, the electrophoretic mobility coefficients $H_{01}$ and $H_{10}$ decrease with increases in $\varphi$ from constants at $\varphi=0$, with an exception that $H_{10}$ may increase with an increase in $\varphi$ when $\kappa a$ is very large. For given values of $\varphi$, $\kappa a$, and $\lambda a$, the coefficient $H_{10}$ is a monotonic decreasing function of $r_{0} / a$ (increasing function of $1-r_{0} / a$ or the relative volume of the porous surface layer of the soft particle) and vanishes at $r_{0} / a=1$ as expected, while $H_{01}$ increases with an increase in $r_{0} / a$ (or relative surface area of the hard core of the soft particle) from zero at $r_{0} / a=0$, but may attain a maximum and then decreases with a further increase in $r_{0} / a$ (or hydrodynamic resistance to the electrophoretic motion of the particle caused by the hard core). The values of $H_{01}$ and $H_{10}$ are comparable for the case of medium $r_{0} / a$ (ca. 1/2). Note that the value of $H_{01}$ always vanishes as $\kappa a \rightarrow \infty$ but the value of $H_{10}$ vanishes as $\kappa a \rightarrow \infty$ only if $\lambda a \rightarrow \infty$.

The second-order coefficients $H_{02}, H_{11}$, and $H_{20}$ for the chemiphoresis of a suspension of soft spheres as functions of $\kappa a, \lambda a, r_{0} / a$, and $\varphi$ are exhibited in Figures 7-9. For given values of $\kappa a, \lambda a$, and $r_{0} / a$, these coefficients (and thus the chemiphoretic mobility) in general first increases with an increase in $\varphi$ from a constant at $\varphi=0$, reaches a maximum, and then decreases with a further increase in $\varphi$. For constant values of $\varphi, \kappa a$ and $\lambda a$, the coefficients $H_{02}, H_{11}$, and $H_{20}$ have the same order of magnitude for the case of $r_{0} / a=0.5$ and about two orders of magnitude less than the coefficients $H_{01}$ and $H_{10}$. The coefficient $H_{02}$ in general increases with an increase in the value of $r_{0} / a$, becomes zero as $r_{0} / a=0$, and does not depend on $\lambda a$ as $r_{0} / a=1$, whereas the coefficient $H_{20}$, in general decreases with an increase in $r_{0} / a$ and equals zero as $r_{0} / a=1$, analogous to the coefficient $H_{10}$. The coefficient $H_{11}$ equals zero in the limits $r_{0} / a=0$ and $r_{0} / a=1$, and thus a maximal value of $H_{11}$ exists between the limits. The location of the maximum shifts to greater $r_{0} / a$ as $\lambda a$ increases, since large volume fraction of the hard cores in the soft particles favors their migration if the resistance to the fluid motion in the surface layers is large. For moderate values of $r_{0} / a$, the three second-order coefficients contribute to the chemiphoretic velocity of the soft spheres comparably. Note that the coefficients $H_{02}, H_{11}$, and $H_{20}$ all vanish as $\kappa a \rightarrow \infty$.

The normalized diffusiophoretic velocity $U / U^{*}$ in a suspension of soft spheres as a function of the scaled fixed charge density $(\kappa a)^{2} \bar{Q}=a^{2} Z e Q / \varepsilon k T$ calculated for the Happel model at $\bar{\sigma}=0, r_{0} / a=0.5$, $\lambda a=1, \varphi=0.1$, and various values of $\kappa a$ is plotted in Figure $10 \mathrm{a}, \mathrm{b}$ for the cases of $\beta=0$ and $\beta=-0.2$, respectively. Similar to the outcomes in Figure 4 for a suspension of hard particles, for specified values of $r_{0} / a, \lambda a, \kappa a$ and $\varphi$, the normalized velocity $U / U^{*}$ for the case of $\beta=0$ (due to the chemiphoresis entirely) is an even function of the fixed charge density and increases monotonically with an increase in $(\kappa a)^{2}|\bar{Q}|$ from zero at $(\kappa a)^{2} \bar{Q}=0$, while $U / U^{*}$ for the case of $\beta=-0.2$ is not necessary a monotonic 
function of $(\kappa a)^{2} \bar{Q}$ and may change its sign twice with the variation of their fixed charge density due to the competition between chemiphoretic and electrophoretic contributions.
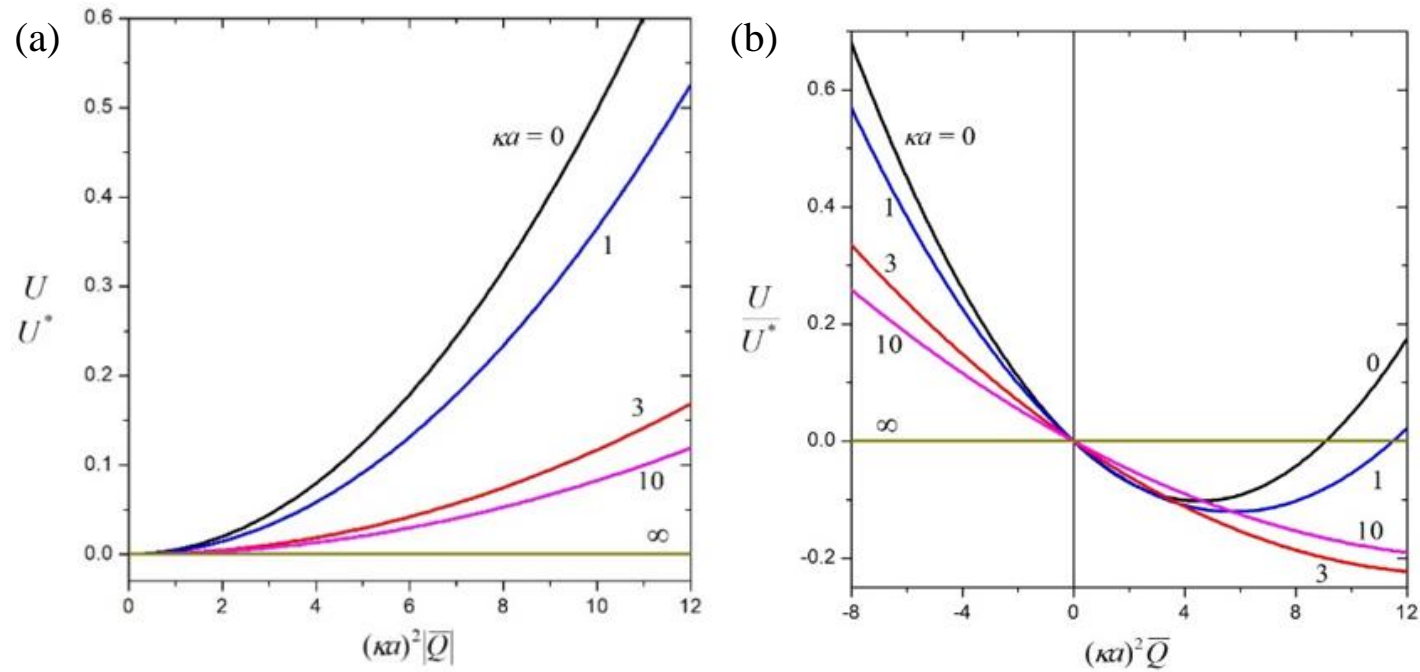

Figure 10. The reduced diffusiophoretic mobility $U / U^{*}$ for a suspension of soft spheres versus the dimensionless fixed charge density $(\kappa a)^{2} \bar{Q}$ at $\bar{\sigma}=0, r_{0} / a=0.5, \lambda a=1, \varphi=0.1$, and various values of $\kappa a$ calculated for the Happel model: (a) $\beta=0$; (b) $\beta=-0.2$.

\section{Conclusions}

The diffusiophoresis of a suspension of charged softs spheres (each is a hard core of surface charge density $\sigma$ covered with a fluid-penetrable porous layer of fixed charge density $Q$ ) in a symmetric electrolyte solution with arbitrary values of the electrokinetic particle radius $k a$, core-to-particle radius ratio $r_{0} / a$, porous-layer shielding parameter $\lambda a$, and particle volume fraction $\varphi$ is analytically studied in this work. Through the use of a unit cell model, the ionic concentration (or electrochemical potential energy), electric potential, and fluid velocity distributions are solved as power expansions in $\sigma$ and $Q$, and an explicit expression for the diffusiophoretic velocity of the soft spheres correct to the second order of $\sigma$ and $Q$ is obtained from a force balance. The effects of the physical characteristics and particle interactions on the diffusiophoresis (including electrophoresis and chemiphoresis) in a suspension of soft particles, which become those of hard particles and porous particles in the limits $r_{0}=a$ and $r_{0}=0$, respectively, are significant and complicated. A similar formula for the electrophoretic velocity of a rigid sphere with low zeta $(\zeta)$ potential was shown to give an excellent approximation for the case of reasonably high zeta potential (with an error of less than $4 \%$ in a $\mathrm{KCl}$ solution for the case of $\zeta e / k T \leq 2)$ [34]. Therefore, our results might be used tentatively for the situation of reasonably high electric potentials or fixed charge densities.

Author Contributions: Conceptualization, H.J.K.; methodology, H.J.K. and W.C.L.; investigation, H.J.K. and W.C.L.; writing—original draft preparation, H.J.K.; writing—review and editing, H.J.K. and W.C.L.; supervision, H.J.K.; funding acquisition, H.J.K. All authors have read and agreed to the published version of the manuscript.

Funding: This research was funded by the Ministry of Science and Technology, Taiwan (Republic of China) grant number MOST 106-2221-E-002-167-MY3.

Conflicts of Interest: The authors declare no conflict of interest. The founding sponsors had no role in the design of the study; in the collection, analyses, or interpretation of data; in the writing of the manuscript, and in the decision to publish the results. 


\section{Appendix A}

Some functions in Section 3 are given here. In Equations (17)-(19),

$$
\begin{gathered}
F_{00 r}(r)=C_{001}+C_{002} \frac{a}{r}+C_{003}\left(\frac{a}{r}\right)^{3}+C_{004}\left(\frac{r}{a}\right)^{2}, \text { if } a<r<b \\
F_{00 r}(r)=C_{005}+\left[C_{006}+C_{007} \alpha_{1}(\lambda r)+C_{008} \beta_{1}(\lambda r)\right]\left(\frac{a}{r}\right)^{3}, \text { if } r_{0}<r<a \\
F_{p 00}(r)=C_{002}\left(\frac{a}{r}\right)^{2}+10 C_{004} \frac{r}{a}, \text { if } a<r<b \\
F_{p 00}(r)=(\lambda a)^{2}\left[-C_{005} \frac{r}{a}+\frac{C_{006}}{2}\left(\frac{a}{r}\right)^{2}\right], \text { if } r_{0}<r<a \\
F_{i j r}(r)=C_{i j 1}-J_{i j}^{(2)}(r)+\left[C_{i j 2}+J_{i j}^{(3)}(r)\right] \frac{a}{r}+\left[C_{i j 3}-\frac{1}{5} J_{i j}^{(5)}(r)\right]\left(\frac{a}{r}\right)^{3} \\
+\left[C_{i j 4}+\frac{1}{5} J_{i j}^{(0)}(r)\right]\left(\frac{r}{a}\right)^{2}, \text { if } a<r<b \\
F_{i j r}(r)=C_{i j 5}+\left[C_{i j 6}+C_{i j 7} \alpha_{1}(\lambda r)+C_{i j 8} \beta_{1}(\lambda r)\right]\left(\frac{a}{r}\right)^{3}-\frac{2}{(\lambda a)^{2}}\left[J_{i j}^{(0)}(r)-\left(\frac{a}{r}\right)^{3} J_{i j}^{(3)}(r)\right. \\
\left.-3 \frac{\beta_{1}(\lambda r)}{(\lambda r)^{3}} J_{i j}^{\alpha}(r)+3 \frac{\alpha_{1}(\lambda r)}{(\lambda r)^{3}} J_{i j}^{\beta}(r)\right], \text { if } r_{0}<r<a \\
F_{p i j}(r)=\left[C_{i j 2}+J_{i j}^{(3)}(r)\right]\left(\frac{a}{r}\right)^{2}+2\left[5 C_{i j 4}+J_{i j}^{(0)}(r)\right] \frac{r}{a}, \text { if } a<r<b \\
C_{p i j}(r)=\lambda_{i j 6}^{2} a r\left[-C_{i j 5}+\frac{a}{2}\left(\frac{3}{r}\right)^{3}\right]+J_{i j}^{(3)}(r)\left(\frac{a}{r}\right)^{2}+2 J_{i j}^{(0)}(r) \frac{r}{a}, \text { if } r_{0}<r<a
\end{gathered}
$$

where the set $(i, j)=(0,1),(1,0),(0,2),(1,1)$, and $(2,0)$,

$$
\begin{gathered}
\alpha_{1}(x)=x \cosh x-\sinh x \\
\beta_{1}(x)=x \sinh x-\cosh x \\
J_{i j}^{(n)}(r)=\int_{a}^{r}\left(\frac{r}{a}\right)^{n} G_{i j}(r) \mathrm{d} r \\
J_{i j}^{\alpha}(r)=\int_{a}^{r} \alpha_{1}(\lambda r) G_{i j}(r) \mathrm{d} r \\
J_{i j}^{\beta}(r)=\int_{a}^{r} \beta_{1}(\lambda r) G_{i j}(r) \mathrm{d} r \\
G_{01}(r)=\frac{\varepsilon \kappa^{2} a^{4}}{3 Z e r} F_{\mu 00}(r) \frac{\mathrm{d} \psi_{\mathrm{eq} 01}}{\mathrm{~d} r} \\
G_{10}(r)=\frac{\varepsilon \kappa^{2} a^{4}}{3 Z e r} F_{\mu 00}(r) \frac{\mathrm{d} \psi_{\mathrm{eq} 10}}{\mathrm{~d} r} \\
G_{02}(r)=-\frac{\varepsilon \kappa^{2} a^{4}}{3 Z e r} W_{01}(r) \frac{\mathrm{d} \psi_{\text {eq } 01}}{\mathrm{~d} r} \\
G_{11}(r)=-\frac{\varepsilon \kappa^{2} a^{4}}{3 Z e r}\left[W_{01}(r) \frac{\mathrm{d} \psi_{\text {eq } 10}}{\mathrm{~d} r}+W_{10}(r) \frac{\mathrm{d} \psi_{\text {eq } 01}}{\mathrm{~d} r}\right] \\
G_{20}(r)=-\frac{\varepsilon \kappa^{2} a^{4}}{3 Z e r} W_{10}(r) \frac{\mathrm{d} \psi_{\text {eq } 10}}{\mathrm{~d} r} \\
W_{i j}(r)=F_{\mu i j}(r)+\frac{Z e}{k T} \psi_{\text {eqij }}(r) F_{\mu 00}(r)
\end{gathered}
$$


In Equations (15), (16), and (A19),

$$
\begin{gathered}
F_{\mu 00}(r)=F_{\psi 00}(r)=\frac{2 r^{3}+r_{0}^{3}}{2 a r^{2} v} \\
F_{\mu i j}(r)=\frac{-1}{6 b r^{2}}\left\{\frac{1}{v b^{3}}\left(2 r^{3}+r_{0}^{3}\right)\left[2 r_{0}^{3} K_{i j}^{(3)}(a, b)+b^{3} K_{i j}^{(0)}(a, b)\right]+\frac{1}{v}\left[2(1-v) r^{3}+r_{0}^{3}\right]\right. \\
\left.\times\left[2 K_{i j}^{(3)}\left(r_{0}, a\right)+K_{i j}^{(0)}\left(r_{0}, a\right)\right]+2 r_{0}^{3} K_{i j}^{(3)}(a, r)-2 r^{3} K_{i j}^{(0)}(a, r)\right\}, \text { if } a \leq r \leq b \\
F_{\mu i j}(r)=\frac{-1}{6 a b r}\left\{\left[2 r_{0}^{3} K_{i j}^{(3)}\left(r_{0}, b\right)+b^{3} K_{i j}^{(0)}\left(r_{0}, b\right)\right] \frac{2 r^{3}+r_{0}^{3}}{v b^{3}}+2 r_{0}^{3} K_{i j}^{(3)}\left(r_{0}, r\right)-2 r^{3} K_{i j}^{(0)}\left(r_{0}, r\right)\right\}, \\
\text { if } r_{0}<r<a \\
F_{\psi i j}(r)=\frac{1}{A \kappa^{2} r^{2}}\left\{A L_{i j}^{\alpha}(r) \beta_{1}(\kappa r)-A L_{i j}^{\beta}(r) \alpha_{1}(\kappa r)\right. \\
+\left[B L_{i j}^{\alpha}\left(r_{0}\right)-C L_{i j}^{\beta}\left(r_{0}\right)\right]\left[\beta_{1}(\kappa b) \alpha_{1}(\kappa r)-\alpha_{1}(\kappa b) \beta_{1}(\kappa r)\right] \\
\left.+L_{i j}^{\alpha}(b) \beta_{1}(\kappa b)\left[E \alpha_{1}(\kappa r)-D \beta_{1}(\kappa r)\right]+L_{i j}^{\beta}(b) \alpha_{1}(\kappa b)\left[B \alpha_{1}(\kappa r)+C \beta_{1}(\kappa r)\right]\right\}
\end{gathered}
$$

where $v=1+r_{0}^{3} / 2 b^{3}$,

$$
\begin{gathered}
K_{i j}^{(n)}\left(r_{1}, r_{2}\right)=\frac{Z e}{k T} \int_{r_{1}}^{r_{2}}\left(\frac{r}{r_{0}}\right)^{n}\left[1-\left(\frac{r_{0}}{r}\right)^{3}\right] \frac{\mathrm{d} \psi_{\mathrm{eq} i j} \mathrm{~d} r}{\mathrm{~d} r} \\
L_{i j}^{\alpha}(r)=\frac{Z e}{k T} \int_{a}^{r} \kappa \alpha_{1}(\kappa r) W_{i j}(r) \mathrm{d} r \\
L_{i j}^{\beta}(r)=\frac{Z e}{k T} \int_{a}^{r} \kappa \beta_{1}(\kappa r) W_{i j}(r) \mathrm{d} r \\
A=\kappa\left(2 b+b \kappa^{2} r_{0}^{2}-2 r_{0}\right) \cosh \left[\kappa\left(b-r_{0}\right)\right]-\left(2+\kappa^{2} r_{0}^{2}-2 \kappa^{2} b r_{0}\right) \sinh \left[\kappa\left(b-r_{0}\right)\right] \\
B=\kappa^{2} r_{0}^{2} \cosh \left(\kappa r_{0}\right)-2 \beta_{1}\left(\kappa r_{0}\right) \\
C=2 \alpha_{1}\left(\kappa r_{0}\right)-\kappa^{2} r_{0}^{2} \sinh \left(\kappa r_{0}\right) \\
D=2 \kappa r_{0} \cosh \left(\kappa r_{0}\right)-N \sinh \left(\kappa r_{0}\right) \\
E=2 \kappa r_{0} \sinh \left(\kappa r_{0}\right)-N \cosh \left(\kappa r_{0}\right)
\end{gathered}
$$

\section{References}

1. Dukhin, S.S.; Derjaguin, B.V. Electrokinetic Phenomena. In Surface and Colloid Science; Matijevic, E., Ed.; Wiley: New York, NY, USA, 1974; Volume 7.

2. Prieve, D.C.; Anderson, J.L.; Ebel, J.P.; Lowell, M.E. Motion of a particle generated by chemical gradients. Part 2. Electrolytes. J. Fluid Mech. 1984, 148, 247-269. [CrossRef]

3. Zhang, X.; Hsu, W.-L.; Hsu, J.-P.; Tseng, S. Diffusiophoresis of a soft spherical particle in a spherical cavity. J. Phys. Chem. B 2009, 113, 8646-8656. [CrossRef] [PubMed]

4. Joo, S.W.; Lee, S.Y.; Liu, J.; Qian, S. Diffusiophoresis of an elongated cylindrical nanoparticle along the axis of a nanopore. ChemPhysChem 2010, 11, 3281-3290. [CrossRef] [PubMed]

5. Keh, H.J. Diffusiophoresis of charged particles and diffusioosmosis of electrolyte solutions. Curr. Opin. Colloid Interface Sci. 2016, 24, 13-22. [CrossRef]

6. Chang, Y.C.; Keh, H.J. Diffusiophoresis of a colloidal cylinder at small finite Péclet numbers. Colloids Interfaces 2019, 3, 44. [CrossRef]

7. Smith, R.E.; Prieve, D.C. Accelerated deposition of latex particles onto a rapidly dissolving steel surface. Chem. Eng. Sci. 1982, 37, 1213-1223. [CrossRef] 
8. Oshanin, G.; Popescu, M.N.; Dietrich, S. Active colloids in the context of chemical kinetics. J. Phys. A Math. Theor. 2017, 50, 134001. [CrossRef]

9. Gupta, A.; Rallabandi, B.; Stone, H.A. Diffusiophoretic and diffusioosmotic velocities for mixtures of valence-asymmetric electrolytes. Phys. Rev. Fluids 2019, 4, 043702. [CrossRef]

10. Hatlo, M.M.; Panja, D.; van Roij, R. Translocation of DNA molecules through nanopores with salt gradients: The role of osmotic flow. Phys. Rev. Lett. 2011, 107, 68101. [CrossRef]

11. Shin, S.; Um, E.; Sabass, B.; Ault, J.T.; Rahimi, M.; Warren, P.B.; Stone, H.A. Size-Dependent control of colloid transport via solute gradients in dead-end channels. Proc. Natl. Acad. Sci. USA 2016, 113, 257-261. [CrossRef]

12. Wilson, J.L.; Shim, S.; Yu, Y.E.; Gupta, A.; Stone, H.A. Diffusiophoresis in Multivalent Electrolytes. Langmuir 2020, 36, 7014-7020. [CrossRef] [PubMed]

13. Anderson, J.L. Colloid transport by interfacial forces. Annu. Rev. Fluid Mech. 1989, 21, 61-99. [CrossRef]

14. Abécassis, B.; Cottin-Bizonne, C.; Ybert, C.; Ajdari, A.; Bocquet, L. Boosting migration of large particles by solute contrasts. Nat. Mater. 2008, 7, 785-789. [CrossRef] [PubMed]

15. Banerjee, A.; Williams, I.; Azevedo, R.N.; Helgeson, M.E.; Squires, T.M. Soluto-Inertial phenomena: Designing long-range, long-lasting, surface-specific interactions in suspensions. Proc. Natl. Acad. Sci. USA 2016, 113, 8612-8617. [CrossRef]

16. Velegol, D.; Garg, A.; Guha, R.; Kar, A.; Kumar, M. Origins of concentration gradients for diffusiophoresis. Soft Matter 2016, 12, 4686-4703. [CrossRef]

17. Pawar, Y.; Solomentsev, Y.E.; Anderson, J.L. Polarization effects on diffusiophoresis in electrolyte gradients. J. Colloid Interface Sci. 1993, 155, 488-498. [CrossRef]

18. Tu, H.J.; Keh, H.J. Particle interactions in diffusiophoresis and electrophoresis of colloidal spheres with thin but polarized double layers. J. Colloid Interface Sci. 2000, 231, 265-282. [CrossRef]

19. Prieve, D.C.; Roman, R. Diffusiophoresis of a rigid sphere through a viscous electrolyte solution. J. Chem. Soc. Faraday Trans. 2 1987, 83, 1287-1306. [CrossRef]

20. Keh, H.J.; Wei, Y.K. Diffusiophoretic mobility of spherical particles at low potential and arbitrary double-layer thickness. Langmuir 2000, 16, 5289-5294. [CrossRef]

21. Wei, Y.K.; Keh, H.J. Diffusiophoretic mobility of charged porous spheres in electrolyte gradients. J. Colloid Interface Sci. 2004, 269, 240-250. [CrossRef]

22. Huang, P.Y.; Keh, H.J. Diffusiophoresis of a spherical soft particle in electrolyte gradients. J. Phys. Chem. B 2012, 116, 7575-7589. [CrossRef] [PubMed]

23. Yeh, Y.Z.; Keh, H.J. Diffusiophoresis of a charged porous shell in electrolyte gradients. Colloid Polym. Sci. 2018, 296, 451-459. [CrossRef]

24. Levine, S.; Neale, G.; Epstein, N. The prediction of electrokinetic phenomena within multiparticle systems II. Sedimentation potential. J. Colloid Interface Sci. 1976, 57, 424-437. [CrossRef]

25. Ohshima, H. Sedimentation potential in a concentrated suspension of spherical colloidal particles. J. Colloid Interface Sci. 1998, 208, 295-301. [CrossRef]

26. Carrique, F.; Arroyo, F.J.; Delgado, A.V. Sedimentation velocity and potential in a concentrated colloidal suspension. Effect of a dynamic Stern layer. Colloids Surfaces A 2001, 195, 157-169. [CrossRef]

27. Ding, J.M.; Keh, H.J. Sedimentation velocity and potential in a suspension of charge-regulating colloidal spheres. J. Colloid Interface Sci. 2001, 243, 331-341. [CrossRef]

28. Keh, H.J.; Chen, W.C. Sedimentation velocity and potential in concentrated suspensions of charged porous spheres. J. Colloid Interface Sci. 2006, 296, 710-720. [CrossRef]

29. Chiu, Y.S.; Keh, H.J. Sedimentation velocity and potential in a concentrated suspension of charged soft spheres. Colloids Surfaces A 2014, 440, 185-196. [CrossRef]

30. Levine, S.; Neale, G.H. The prediction of electrokinetic phenomena within multiparticle systems I. Electrophoresis and electroosmosis. J. Colloid Interface Sci. 1974, 47, 520-529. [CrossRef]

31. Zharkikh, N.I.; Shilov, V.N. Theory of collective electrophoresis of spherical particles in the Henry approximation. Colloid J. USSR Engl. Transl. 1982, 43, 865-870.

32. Ohshima, H. Electrophoretic mobility of spherical colloidal particles in concentrated suspensions. J. Colloid Interface Sci. 1997, 188, 481-485. [CrossRef] 
33. Zholkovskiy, E.K.; Masliyah, J.H.; Shilov, V.N.; Bhattacharjee, S. Electrokinetic phenomena in concentrated disperse systems: General problem formulation and spherical cell approach. Adv. Colloid Interface Sci. 2007, 134, 279-321. [CrossRef] [PubMed]

34. Keh, H.J.; Ding, J.M. Electrophoretic mobility and electric conductivity of suspensions of charge-regulating colloidal spheres. Langmuir 2002, 18, 4572-4583. [CrossRef]

35. Carrique, F.; Arroyo, F.J.; Jimenez, M.L.; Delgado, A.V. Influence of double-layer overlap on the electrophoretic mobility and DC conductivity of a concentrated suspension of spherical particles. J. Phys. Chem. B 2003, 107, 3199-3206. [CrossRef]

36. Huang, H.Y.; Keh, H.J. Electrophoretic mobility and electric conductivity in suspensions of charge-regulating porous particles. Colloid Polym. Sci. 2015, 293, 1903-1914. [CrossRef]

37. Liu, H.C.; Keh, H.J. Electrophoresis and electric conduction in a suspension of charged soft particles. Colloid Polym. Sci. 2016, 294, 1129-1141. [CrossRef]

38. Ohshima, H. Electrical conductivity of a concentrated suspension of spherical colloidal particles. J. Colloid Interface Sci. 1999, 212, 443-448. [CrossRef]

39. Miller, N.P.; Berg, J.C. Experiments on the electrophoresis of porous aggregates. J. Colloid Interface Sci. 1993, 159, 253-254. [CrossRef]

40. Wei, Y.K.; Keh, H.J. Diffusiophoresis in a suspension of spherical particles with arbitrary double-layer thickness. J. Colloid Interface Sci. 2002, 248, 76-87. [CrossRef]

41. Huang, H.Y.; Keh, H.J. Diffusiophoresis in suspensions of charged porous particles. J. Phys. Chem. B 2015, 119, 2040-2050. [CrossRef]

42. Koplik, J.; Levine, H.; Zee, A. Viscosity renormalization in the Brinkman equation. Phys. Fluids 1983, 26, 2864-2870. [CrossRef]

43. Happel, J.; Brenner, H. Low Reynolds Number Hydrodynamics; Nijhoff: Leiden, The Netherlands, 1983.

(C) 2020 by the authors. Licensee MDPI, Basel, Switzerland. This article is an open access article distributed under the terms and conditions of the Creative Commons Attribution (CC BY) license (http://creativecommons.org/licenses/by/4.0/). 